\title{
Spectroscopy of odd-odd nuclei within the interacting boson-fermion-fermion model based on the Gogny energy-density functional
}

\author{
K. Nomura, ${ }^{1,2}$ R. Rodríguez-Guzmán, ${ }^{3}$ and L. M. Robledo ${ }^{4,5}$ \\ ${ }^{1}$ Physics Department, Faculty of Science, University of Zagreb, HR-10000 Zagreb, Croatia \\ ${ }^{2}$ Advanced Science Research Center, Japan Atomic Energy Agency, Tokai, 319-1195 Ibaraki, Japan \\ ${ }^{3}$ Physics Department, Kuwait University, 13060 Kuwait, Kuwait \\ ${ }^{4}$ Departamento de Física Teórica, Universidad Autónoma de Madrid, E-28049 Madrid, Spain \\ ${ }^{5}$ Center for Computational Simulation, Universidad Politécnica de Madrid, Campus de Montegancedo, \\ Boadilla del Monte, E-28660 Madrid, Spain
}

(Received 25 December 2018; published 7 March 2019)

\begin{abstract}
We present a method to calculate spectroscopic properties of odd-odd nuclei within the framework of the interacting boson-fermion-fermion model (IBFFM) based on the Gogny energy-density functional. The $(\beta, \gamma)$-deformation energy surface of the even-even (boson-)core nucleus, spherical single-particle energies, and occupation probabilities of the odd neutron and odd proton are provided by the constrained self-consistent mean-field calculation within the Hartree-Fock-Bogoliubov method with the Gogny-D1M functional. These quantities are used as a microscopic input to fix most of the parameters of the IBFFM Hamiltonian. Only a few coupling constants for the boson-fermion Hamiltonian and the residual neutron-proton interaction are specifically adjusted to reproduce experimental low-energy spectra in odd-mass and odd-odd nuclei, respectively. In this way, the number of free parameters involved in the IBFFM framework is reduced significantly. The method is successfully applied to the description of the low-energy spectra and electromagnetic transition rates in the odd-odd ${ }^{194,196,198} \mathrm{Au}$ nuclei.
\end{abstract}

DOI: 10.1103/PhysRevC.99.034308

\section{INTRODUCTION}

The unified theoretical description of low-lying states in even-even, odd-mass, and odd-odd nuclei is one of the major goals of nuclear structure research. In even-even systems at low energy, nucleons are coupled pairwise and the type of couplings determines the low-lying collective structure of vibrational and rotational states. The microscopic description of low-lying collective states in even-even systems has been extensively pursued with numerous theoretical methods [1-6]. However, the description of odd-mass and odd-odd nuclei is more cumbersome, due to the fact that in those systems both collective and single-particle motions have to be treated on the same footing [1,7].

The interacting boson model (IBM) [3] has been remarkably successful in the phenomenological study of low-lying structures in medium-mass and heavy even-even nuclei. In its simplest version, the building blocks of the IBM are the monopole $s$ and quadrupole $d$ bosons, which represent the collective pairs of valence nucleons coupled to spin and parity $0^{+}$and $2^{+}$, respectively $[3,8]$. The microscopic foundation of the IBM starting from the nucleonic degrees of freedom has been extensively pursued in the literature [8-13]. In particular, a systematic method of deriving the IBM Hamiltonian from microscopic input was developed in [12]. In this approach, the deformation energy surface that is obtained from the selfconsistent mean-field (SCMF) calculation based on a given energy-density functional (EDF) is mapped onto the expectation value of the IBM Hamiltonian in the boson coherent state [14]. This procedure completely determines the strength parameters of the IBM Hamiltonian. Since the EDF framework allows for a global mean-field description of intrinsic properties of nuclei over the entire Segré's chart, it has become possible to determine in a unified way the parameters of the IBM Hamiltonian basically for any arbitrary nucleus.

The method mentioned above was recently extended to odd-mass systems [15] by considering the coupling between bosonic (collective) degrees of freedom and an unpaired nucleon within the framework of the Interacting boson-fermion model (IBFM) [16]. In this extension, the even-even core (IBM) Hamiltonian, the single-particle energies, and occupation probabilities of the odd particle, which are building blocks of the IBFM Hamiltonian, have been completely determined based on the output of a SCMF calculation. Even though a few strength parameters for the particle-boson coupling are treated as free parameters, the method allows for an accurate, systematic, and computationally feasible description of various low-energy properties of odd-mass, medium-mass, and heavy nuclei: e.g., signatures of shape phase transitions [17-20], octupole correlations in neutron-rich odd-mass $\mathrm{Ba}$ isotopes [21], and the structure of neutron-rich odd-mass $\mathrm{Kr}$ isotopes [22].

In this work, we extend these studies to odd-odd nuclei by using the interacting boson-fermion-fermion model (IBFFM) [16,23]. The IBFFM is an extension of the IBFM that considers odd-odd nuclei as a system composed of an IBM core plus an unpaired neutron and an unpaired proton. 
The IBM-core and particle-boson coupling Hamiltonians are determined in a way similar to that employed for odd-mass nuclei [15]. The only additional parameters are the coefficients of the residual neutron-proton interaction. They are determined to reasonably reproduce the experimental data for the low-lying spectra of the considered odd-odd nuclei. The microscopic input used to determine part of the IBFFM Hamiltonian is obtained by constrained SCMF calculations within the Hartree-Fock-Bogoliubov (HFB) method based on the Gogny D1M EDF [24]. The two most relevant parametrizations of the finite range Gogny force, namely D1S [25] and D1M [24], have proved through the years to provide a reliable description of many collective phenomena all over the periodic table (see [26,27] for some examples). Our choice of D1M is based solely on its better performance to describe binding energies.

As an application of the proposed methodology, we specifically study the properties of the odd-odd ${ }^{194,196,198}$ Au nuclei. Their low-lying structures are described by unpaired neutron and proton holes coupled with the even-even core nuclei ${ }_{196,198,200} \mathrm{Hg}$. The IBM parameters for the even-even cores were already obtained in Ref. [28] as part of a comprehensive study of shape coexistence and low-lying structures in the entire $\mathrm{Hg}$ isotopic chain within the configuration-mixing IBM method based on the Gogny-D1M EDF. The results obtained suggest that the nuclei ${ }^{196,198,200} \mathrm{Hg}$ have weakly oblate deformed to nearly spherical ground-state shapes. For the neighboring odd- $N$ nuclei ${ }^{195,197,199} \mathrm{Hg}$ and odd- $Z$ nuclei ${ }^{195,197,199} \mathrm{Au}$, there are plenty of experimental data to determine the boson-fermion strength parameters. In addition, the odd-odd Au nuclei in this mass region have previously been extensively studied within the IBFFM framework: e.g., by means of numerical studies $[29,30]$, or by pure-algebraic approaches [31-33] in the context of nuclear supersymmetry [34]. Those results will be a good reference to compare with our less phenomenological results.

On the other hand, it is worthwhile to mention that microscopic nuclear structure models are also applied in the spectroscopic studies of odd-mass and/or odd-odd nuclei with the Gogny force. As an example, let us mention the studies of various low-energy properties of odd-mass systems at the mean-field level using full blocking [35] or the equal filling approximation [36-38]. To our knowledge there is only one study [39] of odd-odd nuclei focused on the ability to reproduce the empirical Gallagher-Moszkowski (GM) rule. As shown in this reference, the GM rule is not fulfilled by the Gogny force and the failure is traced back to the lack of additional proton-neutron interaction terms in the interaction. This difficulty and the inability of any effective interaction to reach spectroscopic accuracy for the spectra of odd nuclei [40] point to the necessity of adding extra terms with extra parameters that can be fitted locally to improve the quality of the description of odd and odd-odd nuclei. This is achieved in our model through the set of extra terms added with parameters not fixed by the EDF input. Another source of difficulties hampering to reach spectroscopic accuracy in the description of odd nuclei with EDFs is the impact of dynamical correlations such as those coming from symmetry restoration [2]. In the last few years it has been possible to include time-reversal symmetry and blocking effects along with angular momentum and particle number projection [41], but the complexity of the problem prevents its use beyond very light systems like ${ }^{24} \mathrm{Mg}[41,42]$.

This paper is organized as follows: In Sec. II we describe the procedure to construct the IBFFM Hamiltonian based on the SCMF calculation. In Sec. III, the spectroscopic properties of the even-even $\mathrm{Hg}$ nuclei are briefly reviewed. In the same section, the results for low-energy spectra in the odd- $N \mathrm{Hg}$ and the odd- $Z \mathrm{Au}$ isotopes are discussed, followed by the results of the spectroscopic calculations for the odd-odd Au nuclei. Finally, a short summary and concluding remarks are given in Sec. IV.

\section{THEORETICAL FRAMEWORK}

\section{A. Hamiltonian}

In this work we use the version of the IBFFM that distinguishes between neutron and proton degrees of freedom (denoted hereafter as IBFFM-2). The IBFFM-2 Hamiltonian is expressed as

$$
\hat{H}=\hat{H}_{\mathrm{B}}+\hat{H}_{\mathrm{F}}^{v}+\hat{H}_{\mathrm{F}}^{\pi}+\hat{H}_{\mathrm{BF}}^{v}+H_{\mathrm{BF}}^{\pi}+\hat{V}_{\mathrm{res}} .
$$

The first term in Eq. (1) is the neutron-proton IBM (IBM2) Hamiltonian [8] that describes the even-even core nuclei ${ }_{196,198,200} \mathrm{Hg}$. The second and third terms represent the Hamiltonian for an odd neutron and an odd proton, respectively. The fourth and fifth terms correspond to the interaction Hamiltonians describing the couplings of the odd neutron and of the odd proton to the IBM-2 core, respectively. The last term in Eq. (1) is the residual interaction between the odd neutron and odd proton.

For the boson-core Hamiltonian $\hat{H}_{\mathrm{B}}$ the standard IBM-2 Hamiltonian is adopted:

$$
\hat{H}_{\mathrm{B}}=\epsilon\left(\hat{n}_{d_{v}}+\hat{n}_{d_{\pi}}\right)+\kappa \hat{Q}_{\nu} \cdot \hat{Q}_{\pi}+\kappa^{\prime} \hat{L} \cdot \hat{L},
$$

where $\hat{n}_{d_{\rho}}=d_{\rho}^{\dagger} \cdot \tilde{d}_{\rho}(\rho=v, \pi)$ is the $d$-boson number operator, $\hat{Q}_{\rho}=d_{\rho}^{\dagger} s_{\rho}+s_{\rho}^{\dagger} \tilde{d}_{\rho}^{\dagger}+\chi_{\rho}\left(d_{\rho}^{\dagger} \times \tilde{d}_{\rho}\right)^{(2)}$ is the quadrupole operator, and $\hat{L}=\hat{L}_{v}+\hat{L}_{\pi}$ is the angular momentum operator with $\hat{L}_{\rho}=\sqrt{10}\left(d_{\rho}^{\dagger} \times \tilde{d}_{\rho}\right)^{(1)}$. The different parameters of the Hamiltonian are denoted by $\epsilon, \kappa, \chi_{\nu}, \chi_{\pi}$, and $\kappa^{\prime}$. The doubly magic nucleus ${ }^{208} \mathrm{~Pb}$ is taken as the inert core for the boson space. The numbers of neutron $N_{v}$ and proton $N_{\pi}$ bosons equal the number of neutron-hole and proton-hole pairs, respectively. As a consequence, $N_{\pi}=1$ and $N_{v}=5,4$, and 3 for the ${ }^{196,198,200} \mathrm{Hg}$ nuclei, respectively.

The Hamiltonian for the odd nucleon reads

$$
\hat{H}_{\mathrm{F}}^{\rho}=-\sum_{j_{\rho}} \epsilon_{j_{\rho}} \sqrt{2 j_{\rho}+1}\left(a_{j_{\rho}}^{\dagger} \times \tilde{a}_{j_{\rho}}\right)^{(0)}
$$

with $\epsilon_{j_{\rho}}$ being the single-particle energy of the odd nucleon. $j_{\nu}\left(j_{\pi}\right)$ stands for the angular momentum of the odd neutron (proton). $a_{j_{\rho}}^{(\dagger)}$ represents the fermion annihilation (creation) operator and $\tilde{a}_{j_{\rho}}$ is defined as $\tilde{a}_{j m}=(-1)^{j-m} a_{j-m}$. For the fermion valence space, we consider the full neutron major shell $N=82-126$, i.e., $3 p_{1 / 2}, 3 p_{3 / 2}, 2 f_{5 / 2}, 2 f_{7 / 2}, 1 h_{9 / 2}$, and $1 i_{13 / 2}$ orbitals, and the full proton major shell $Z=50-82$, i.e., $3 s_{1 / 2}, 2 d_{3 / 2}, 2 d_{5 / 2}, 1 g_{7 / 2}$, and $1 h_{11 / 2}$ orbitals. 
For the boson-fermion interaction term $\hat{H}_{\mathrm{BF}}^{\rho}$ in Eq. (1), we use the following form

$$
\hat{H}_{\mathrm{BF}}^{\rho}=\Gamma_{\rho} \hat{Q}_{\rho^{\prime}} \cdot \hat{q}_{\rho}+\Lambda_{\rho} \hat{V}_{\rho^{\prime} \rho}+A_{\rho} \hat{n}_{d_{\rho}} \hat{n}_{\rho},
$$

where $\rho^{\prime} \neq \rho$, and the first, second, and third terms are the quadrupole dynamical, exchange, and monopole terms, respectively. The parameters of the interaction Hamiltonian are denoted by $\Gamma_{\rho}, \Lambda_{\rho}$, and $A_{\rho}$. As in the previous studies [43,44], we assume that both the dynamical and exchange terms are dominated by the interaction between unlike particles (i.e., between the odd neutron and proton bosons and between the odd proton and neutron bosons). We also assume that for the monopole term the interaction between like particles (i.e., between the odd neutron and neutron bosons and between the odd proton and proton bosons) plays a dominant role. In Eq. (4) $\hat{Q}_{\rho}$ is the same bosonic quadrupole operator as in the IBM-2 Hamiltonian in Eq. (2). The fermionic quadrupole operator $\hat{q}_{\rho}$ reads

$$
\hat{q}_{\rho}=\sum_{j_{\rho} j_{\rho}^{\prime}} \gamma_{j_{\rho} j_{\rho}^{\prime}}\left(a_{j_{\rho}}^{+} \times \tilde{a}_{j_{\rho}^{\prime}}\right)^{(2)},
$$

where $\quad \gamma_{j_{\rho} j_{\rho}^{\prime}}=\left(u_{j_{\rho}} u_{j_{\rho}^{\prime}}-v_{j_{\rho}} v_{j_{\rho}^{\prime}}\right) Q_{j_{\rho} j_{\rho}^{\prime}} \quad$ and $\quad Q_{j_{\rho} j_{\rho}^{\prime}}=$ $\left\langle l \frac{1}{2} j_{\rho} \| Y^{(2)}|| l^{\prime} \frac{1}{2} j_{\rho}^{\prime}\right\rangle$ represents the matrix element of the fermionic quadrupole operator in the considered single-particle basis. The exchange term $\hat{V}_{\rho^{\prime} \rho}$ in Eq. (4) reads

$$
\begin{aligned}
\hat{V}_{\rho^{\prime} \rho}= & -\left(s_{\rho^{\prime}}^{+} \tilde{d}_{\rho^{\prime}}\right)^{(2)}\left\{\sum_{j_{\rho} j_{\rho}^{\prime} j_{\rho}^{\prime \prime}} \sqrt{\frac{10}{N_{\rho}\left(2 j_{\rho}+1\right)}} \beta_{j_{\rho} j_{\rho}^{\prime}} \beta_{j_{\rho}^{\prime \prime} j_{\rho}}\right. \\
& \left.\left.:\left(\left(d_{\rho}^{+} \times \tilde{a}_{j_{\rho}^{\prime \prime}}\right)^{\left(j_{\rho}\right)} \times\left(a_{j_{\rho}^{\prime}}^{+} \times \tilde{s}_{\rho}\right)^{\left(j_{\rho}^{\prime}\right)}\right)^{(2)}:\right\}+ \text { (H.c. }\right),
\end{aligned}
$$

with $\beta_{j_{\rho} j_{\rho}^{\prime}}=\left(u_{j_{\rho}} v_{j_{\rho}^{\prime}}+v_{j_{\rho}} u_{j_{\rho}^{\prime}}\right) Q_{j_{\rho} j_{\rho}^{\prime}}$. In the second line of the above equation the notation : $(\cdots)$ : indicates normal ordering. In the monopole interactions, the number operator for the odd fermion is expressed as $\hat{n}_{\rho}=\sum_{j_{\rho}}\left(-\sqrt{2 j_{\rho}+1}\right)\left(a_{j_{\rho}}^{+} \times\right.$ $\left.\tilde{a}_{j_{\rho}}\right)^{(0)}$.

In previous IBFFM calculations [45,46], the residual interaction $\hat{V}_{\text {res }}$ in Eq. (1) contained a quadrupole-quadrupole, delta, spin-spin-delta, spin-spin, and tensor interaction. However, we find that only the delta and spin-spin-delta terms are enough to provide a good description of the low-lying states in the odd-odd nuclei considered here. Therefore, the residual interaction used here reads

$$
\hat{V}_{\text {res }}=4 \pi \delta\left(\mathbf{r}_{v}-\mathbf{r}_{\pi}\right)\left(u_{0}+u_{1} \sigma_{v} \cdot \sigma_{\pi}\right),
$$

with $u_{0}$ and $u_{1}$ the parameters. Furthermore, the matrix element of the residual interaction $\hat{V}_{\text {res }}$, denoted by $V_{\text {res }}^{\prime}$, can be expressed as [46]

$$
\begin{aligned}
V_{\mathrm{res}}^{\prime}= & \left(u_{j_{v}^{\prime}} u_{j_{\pi}^{\prime}} u_{j_{v}} u_{j_{v}}+v_{j_{v}^{\prime}} v_{j_{\pi}^{\prime}} v_{j_{v}} v_{j_{v}}\right) V_{j_{v}^{\prime} j_{\pi}^{\prime} j_{v} j_{\pi}}^{J} \\
& -\left(u_{j_{v}^{\prime}} v_{j_{\pi}^{\prime}} u_{j_{v}} v_{j_{\pi}}+v_{j_{v}^{\prime}} u_{j_{\pi}^{\prime}} v_{j_{v}} u_{j_{\pi}}\right) \\
& \times \sum_{J^{\prime}}\left(2 J^{\prime}+1\right)\left\{\begin{array}{lll}
j_{v}^{\prime} & j_{\pi} & J^{\prime} \\
j_{v} & j_{\pi}^{\prime} & J
\end{array}\right\} V_{j_{v}^{\prime} j_{\pi} j_{v} j_{\pi}^{\prime}}^{J^{\prime}},
\end{aligned}
$$

where

$$
V_{j_{\nu}^{\prime} j_{\pi}^{\prime} j_{v} j_{\pi}}^{J}=\left\langle j_{v}^{\prime} j_{\pi}^{\prime} ; J\left|\hat{V}_{\mathrm{res}}\right| j_{v} j_{\pi} ; J\right\rangle
$$

is the matrix element between the neutron-proton pairs, and $J$ stands for the total angular momentum of the neutron-proton pair. The bracket in Eq. (8) stands for the Racah coefficient. Also in Eq. (8) the terms resulting from contractions are ignored as in Ref. [47]. A similar residual neutron-proton interaction is used in the two-quasiparticle rotor-model calculation in Ref. [48].

\section{B. Procedure to build the IBFFM-2 Hamiltonian}

The ingredients of the IBFFM-2 Hamiltonian $\hat{H}$ in Eq. (1) are determined with the following procedure.

(1) First, the IBM-2 Hamiltonian is determined by using the methods of Refs. [12,13]: the $(\beta, \gamma)$-deformation energy surface obtained from the constrained GognyD1M HFB calculation is mapped onto the expectation value of the IBM-2 Hamiltonian in the boson coherent state [14]. This procedure completely determines the parameters $\epsilon, \kappa, \chi_{\nu}$, and $\chi_{\pi}$ in the IBM-2 Hamiltonian. Only the strength parameter $\kappa^{\prime}$ for the $\hat{L} \cdot \hat{L}$ term is determined separately from the other parameters, by adjusting the cranking moment of inertia in the boson intrinsic state to the corresponding ThoulessValatin [49] moment of inertia obtained by the GognyHFB SCMF calculation at the equilibrium mean-field minimum [13].

(2) Second, the strength parameters for the boson-fermion coupling Hamiltonians $\hat{H}_{\mathrm{BF}}^{v}$ and $\hat{H}_{\mathrm{BF}}^{\pi}$ for the odd $-N \mathrm{Hg}$ and odd $Z$ Au nuclei, respectively, is determined by using the procedure of [15]: Single-particle energies and occupation probabilities of the odd nucleon are provided by the Gogny-HFB calculation constrained to zero deformation (see Ref. [50] for details); optimal values of the parameters $\Gamma_{\nu}, \Lambda_{\nu}$, and $A_{v}\left(\Gamma_{\pi}, \Lambda_{\pi}\right.$, and $A_{\pi}$ ), are chosen separately for positive and negative parity, so as to reproduce the experimental low-energy levels of each of the considered odd- $N \mathrm{Hg}$ (odd- $Z \mathrm{Au}$ ) nuclei.

(3) By following previous IBFFM calculations $[23,45,46]$, the same strength parameters $\Gamma_{v}, \Lambda_{v}$, and $A_{v}\left(\Gamma_{\pi}, \Lambda_{\pi}\right.$, and $A_{\pi}$ ) as those obtained for the odd- $N \mathrm{Hg}$ (odd- $Z$ $\mathrm{Au}$ ) nuclei in the previous step are used for the oddodd nuclei. The single-particle energies and occupation probabilities are, however, newly calculated for the odd-odd systems.

(4) Finally, the parameters in the residual interaction $\hat{V}_{\text {res }}$, i.e., $u_{0}$ and $u_{1}$, are determined so as to reasonably reproduce the low-lying spectra in the studied oddodd nuclei. The fixed values $u_{0}=-0.3 \mathrm{MeV}$ and $u_{1}=-0.033 \mathrm{MeV}$ for positive parity, and $u_{0}=-0.3$ $\mathrm{MeV}$ and $u_{1}=0.0 \mathrm{MeV}$ for negative-parity states, are adopted. The ratio, $u_{0} / u_{1} \approx 9$, was also considered in [48].

The values of the IBM-2 parameters employed in the present work are shown in Table I. They are exactly the same 
TABLE I. The adopted parameters of the IBM-2 Hamiltonian $\hat{H}_{\mathrm{B}}$ in Eq. (2). Theyz are taken from Ref. [28].

\begin{tabular}{cccccc}
\hline \hline & $\epsilon(\mathrm{MeV})$ & $\kappa(\mathrm{MeV})$ & $\chi_{\nu}$ & $\chi_{\pi}$ & $\kappa^{\prime}(\mathrm{MeV})$ \\
\hline${ }^{196} \mathrm{Hg}$ & 0.710 & -0.517 & 0.836 & 0.613 & 0.0041 \\
${ }^{198} \mathrm{Hg}$ & 0.675 & -0.470 & 1.333 & 0.166 & 0.0043 \\
${ }^{196} \mathrm{Hg}$ & 0.636 & -0.328 & 0.891 & 0.684 & 0.0018 \\
\hline \hline
\end{tabular}

as those used in Ref. [28]. The fitted strength parameters for the Hamiltonian $\hat{H}_{\mathrm{BF}}^{v}\left(\hat{H}_{\mathrm{BF}}^{\pi}\right)$, i.e., $\Gamma_{\nu}, \Lambda_{\nu}$, and $A_{v}\left(\Gamma_{\pi}, \Lambda_{\pi}\right.$, and $A_{\pi}$ ) are shown in Table II (Table III). The fixed value $\Gamma_{\rho}=0.8 \mathrm{MeV}$ is used for the strength parameter for the quadrupole dynamical term for all the odd-mass and odd-odd nuclei and for both parities. Other parameters do not differ too much between neighboring isotopes. Tables IV, V, and VI summarize the single-particle energies and occupation probabilities obtained from the Gogny-HFB SCMF calculations for the studied odd $-N \mathrm{Hg}$, odd- $Z \mathrm{Au}$, and odd-odd $\mathrm{Au}$ isotopes, respectively. We note that the single-particle energies and occupation probabilities for the odd- $N \mathrm{Hg}$ (Table IV) and odd$\mathrm{Z}$ Au (Table V) nuclei are almost identical to those computed for the odd-odd Au nuclei (see Table VI).

Once all the parameters of the IBFFM-2 Hamiltonian are obtained, it is diagonalized numerically in the basis $\left|L_{v} L_{\pi}(L) ; j_{\nu} j_{\pi}(J): I\right\rangle$, using the computer program TWBOS [51]. $L_{v}\left(L_{\pi}\right)$ and $L$ are the angular momentum for neutron (proton) bosons and the total angular momentum for the eveneven boson core, respectively. Finally, I stands for the total angular momentum of the coupled system.

\section{Transition operators}

Using the eigenstates of the IBFFM-2 Hamiltonian, we can determine the electric quadrupole $(E 2)$ and magnetic dipole $(M 1)$ properties of the odd-odd nuclei. In the present framework, the $E 2$ operator $\hat{T}^{(E 2)}$ takes the following form:

$$
\begin{aligned}
\hat{T}^{(E 2)}= & e_{\nu}^{B} \hat{Q}_{\nu}+e_{\pi}^{B} \hat{Q}_{\pi}-\frac{1}{\sqrt{5}} \sum_{\rho=\nu, \pi} \sum_{j_{\rho} j_{\rho}^{\prime}}\left(u_{j_{\rho}} u_{j_{\rho}^{\prime}}-v_{j_{\rho}} v_{j_{\rho}^{\prime}}\right) \\
& \times\left\langle j_{\rho}^{\prime}\left\|e_{\rho}^{F} r^{2} Y^{(2)}\right\| j_{\rho}\right\rangle\left(a_{j_{\rho}}^{\dagger} \times \tilde{a}_{j_{\rho}^{\prime}}\right)^{(2)}
\end{aligned}
$$

where $e_{\rho}^{B}$ and $e_{\rho}^{F}$ stand for the effective charges for the boson and fermion systems, respectively. The fixed values $e_{v}^{B}=$ $e_{\pi}^{B}=0.15 \mathrm{eb}$, which are taken from Ref. [28], and $e_{v}^{F}=0.5$ $e \mathrm{~b}$ and $e_{\pi}^{F}=1.5 e \mathrm{~b}$ are used.

TABLE II. Strength parameters of the Hamiltonian $\hat{H}_{\mathrm{BF}}^{v}$ (in MeV) employed for the odd- $N$ nuclei ${ }^{195,197,199} \mathrm{Hg}$ and odd-odd nuclei ${ }_{194,196,198} \mathrm{Au}$.

\begin{tabular}{ccccccc}
\hline \hline & $\Gamma_{v}^{+}$ & $\Lambda_{v}^{+}$ & $A_{v}^{+}$ & $\Gamma_{v}^{-}$ & $\Lambda_{v}^{-}$ & $A_{v}^{-}$ \\
\hline${ }^{194} \mathrm{Au},{ }^{195} \mathrm{Hg}$ & 0.80 & 0.0 & -0.10 & 0.80 & 2.00 & -0.80 \\
${ }^{196} \mathrm{Au},{ }^{197} \mathrm{Hg}$ & 0.80 & 0.0 & 0.0 & 0.80 & 1.50 & -0.40 \\
${ }^{198} \mathrm{Au},{ }^{199} \mathrm{Hg}$ & 0.80 & 0.0 & -0.20 & 0.80 & 1.20 & -0.35 \\
\hline \hline
\end{tabular}

TABLE III. Strength parameters of the Hamiltonian $\hat{H}_{\mathrm{BF}}^{\pi}$ (in $\mathrm{MeV}$ ) employed for the odd- $Z$ nuclei ${ }^{195,197,199} \mathrm{Au}$ and odd-odd nuclei ${ }_{194,196,198} \mathrm{Au}$.

\begin{tabular}{ccccccc}
\hline \hline & $\Gamma_{\pi}^{+}$ & $\Lambda_{\pi}^{+}$ & $A_{\pi}^{+}$ & $\Gamma_{\pi}^{-}$ & $\Lambda_{\pi}^{-}$ & $A_{\pi}^{-}$ \\
\hline${ }^{194,195} \mathrm{Au}$ & 0.80 & 1.50 & 0.0 & 0.80 & 1.50 & -0.80 \\
${ }^{196,197} \mathrm{Au}$ & 0.80 & 1.60 & 0.0 & 0.80 & 0.00 & 0.0 \\
${ }^{198,199} \mathrm{Au}$ & 0.80 & 2.40 & 0.0 & 0.80 & 0.00 & 0.0 \\
\hline \hline
\end{tabular}

The $M 1$ transition operator $\hat{T}^{(M 1)}$ reads:

$$
\begin{aligned}
\hat{T}^{(M 1)} & \sqrt{\frac{3}{4 \pi}}\left\{g_{\nu}^{B} \hat{L}_{v}^{B}+g_{\pi}^{B} \hat{L}_{\pi}^{B}-\frac{1}{\sqrt{3}} \sum_{\rho=\nu, \pi} \sum_{j j^{\prime}}\left(u_{j_{\rho}} u_{j_{\rho}^{\prime}}+v_{j_{\rho}} v_{j_{\rho}^{\prime}}\right)\right. \\
& \left.\times\left\langle j_{\rho}^{\prime}|| g_{l}^{\rho} \mathbf{l}+g_{s}^{\rho} \mathbf{s}|| j_{\rho}\right\rangle\left(a_{j_{\rho}}^{\dagger} \times \tilde{a}_{j_{\rho}^{\prime}}\right)^{(1)}\right\} .
\end{aligned}
$$

In this expression, $g_{v}^{B}$ and $g_{\pi}^{B}$ are the $g$ factors for the neutron and proton bosons, respectively. The fixed values $g_{v}^{B}=0 \mu_{N}$ and $g_{\pi}^{B}=1.0 \mu_{N}[3,52]$ are used in this work. For the neutron (proton) $g$ factors, the usual Schmidt values $g_{l}^{v}=0 \mu_{N}$ and $g_{s}^{v}=-3.82 \mu_{N}\left(g_{l}^{\pi}=1.0 \mu_{N}\right.$ and $\left.g_{s}^{\pi}=5.58 \mu_{N}\right)$ are used. The $g_{s}$ value for both the proton and neutron are quenched by $30 \%$.

We note that the forms of the operators $\hat{T}^{(E 2)}$ [Eq. (10)] and $\hat{T}^{(M 1)}$ [Eq. (11)] have been used in previous IBFFM-2 calculations $[23,45,46]$.

As we show later, we have computed the $B(E 2)$ and $B(M 1)$ transition rates, the spectroscopic quadrupole moment $Q(I)$, and the magnetic moment $\mu(I)$ for the odd-odd nuclei 194,196,198 $\mathrm{Au}$, using the computer code TWBTRN [51].

\section{RESULTS AND DISCUSSION}

\section{A. Even-even $\mathrm{Hg}$ isotopes}

In this section, we briefly discuss relevant results for the even-even nuclei ${ }^{196,198,200} \mathrm{Hg}$, which were already presented in Ref. [28]. We plot in Fig. 1 the Gogny-D1M and mapped IBM-2 energy surfaces for the ${ }^{196,198,200} \mathrm{Hg}$ nuclei. In Fig. 1, the Gogny-D1M energy surface for the ${ }^{196} \mathrm{Hg}$ nucleus exhibits a single oblate minimum located at $\beta \approx 0.13$. The oblate minimum becomes less pronounced in ${ }^{198} \mathrm{Hg}$ and, finally, the ${ }^{200} \mathrm{Hg}$ nucleus exhibits a near spherical shape with a very

TABLE IV. Neutron single-particle energies $\epsilon_{j_{v}}$ (in $\mathrm{MeV}$ ) and occupation probabilities $v_{j_{T}}^{2}$ used in the present study for the odd- $N$ nuclei ${ }^{195,197,199} \mathrm{Hg}$.

\begin{tabular}{llllllll}
\hline \hline & & $3 p_{1 / 2}$ & $3 p_{3 / 2}$ & $2 f_{5 / 2}$ & $2 f_{7 / 2}$ & $1 h_{9 / 2}$ & $1 i_{13 / 2}$ \\
\hline${ }^{195} \mathrm{Hg}$ & $\epsilon_{j_{v}}$ & 0.000 & 0.921 & 1.033 & 3.819 & 4.283 & 1.537 \\
& $v_{j_{v}}^{2}$ & 0.248 & 0.515 & 0.554 & 0.944 & 0.951 & 0.702 \\
${ }^{197} \mathrm{Hg}$ & $\epsilon_{j_{v}}$ & 0.000 & 0.937 & 1.056 & 3.846 & 4.366 & 1.570 \\
& $v_{j_{v}}^{2}$ & 0.289 & 0.590 & 0.631 & 0.956 & 0.962 & 0.769 \\
${ }^{199} \mathrm{Hg}$ & $\epsilon_{j_{v}}$ & 0.000 & 0.957 & 1.078 & 3.877 & 4.449 & 1.605 \\
& $v_{j_{v}}^{2}$ & 0.338 & 0.670 & 0.713 & 0.967 & 0.973 & 0.834 \\
\hline \hline
\end{tabular}


TABLE V. Proton single-particle energies $\epsilon_{j_{\pi}}$ and occupation probabilities $v_{j_{\pi}}^{2}$ used in the present study for the odd- $Z$ nuclei ${ }_{195,197,199} \mathrm{Au}$.

\begin{tabular}{lllllll}
\hline \hline & & $3 s_{1 / 2}$ & $2 d_{3 / 2}$ & $2 d_{5 / 2}$ & $1 g_{7 / 2}$ & $1 h_{11 / 2}$ \\
\hline${ }^{195} \mathrm{Au}$ & $\epsilon_{j_{\pi}}$ & 0.000 & 0.907 & 2.624 & 5.163 & 0.840 \\
& $v_{j_{\pi}}^{2}$ & 0.617 & 0.870 & 0.968 & 0.989 & 0.864 \\
${ }^{197} \mathrm{Au}$ & $\epsilon_{j_{\pi}}$ & 0.00 & 0.888 & 2.592 & 5.153 & 0.834 \\
& $v_{j_{\pi}}^{2}$ & 0.619 & 0.869 & 0.968 & 0.989 & 0.865 \\
${ }^{199} \mathrm{Au}$ & $\epsilon_{j_{\pi}}$ & 0.000 & 0.865 & 2.559 & 5.133 & 0.817 \\
& $v_{j_{\pi}}^{2}$ & 0.624 & 0.867 & 0.967 & 0.989 & 0.864 \\
\hline \hline
\end{tabular}

shallow oblate minimum at $\beta \approx 0.08$. The mapped IBM-2 energy surfaces on the right-hand side in Fig. 1 reproduce the basic features of the original Gogny-D1M ones around the global minimum, but look rather flat in the region away from the minimum. This is due to the restricted boson model space [12], which only comprises a finite number of bosons.

The calculated and experimental [53] low-lying spectra are shown in Fig. 2. The yrast levels for all the considered even-even nuclei are described reasonably well. However, the theoretical energy levels, in particular for ${ }^{196,198} \mathrm{Hg}$, look more stretched than the experimental ones. For instance, our calculation is not able to account for the excitation energy of the low-lying $0_{3}^{+}$level of ${ }^{196,198} \mathrm{Hg}$. This discrepancy could be remedied by including in the IBM-2 model space the intruder configurations that are associated with coexisting mean-field minima. These configurations are, however, not considered for the nuclei studied here [28], since their corresponding Gogny-D1M energy surfaces only exhibit a single mean-field minimum (see Fig. 1).

\section{B. Odd-mass $\mathrm{Hg}$ and Au isotopes}

Next, we discuss the spectroscopic properties of the odd-mass nuclei, obtained within the neutron-proton IBFM (IBFM-2). For the diagonalization of the IBFM-2 Hamiltonian, the computer code PBOS is used. The theoretical and experimental low-energy spectra for the odd- $N$ nuclei ${ }^{195,197,199} \mathrm{Hg}$ are compared in Fig. 3. Especially for the positive-parity states, which are based on the unique-parity $v i_{13 / 2}$ configuration, the present calculation provides an excellent description of the experimental spectra for the considered odd- $N$ nuclei, although only three parameters are involved

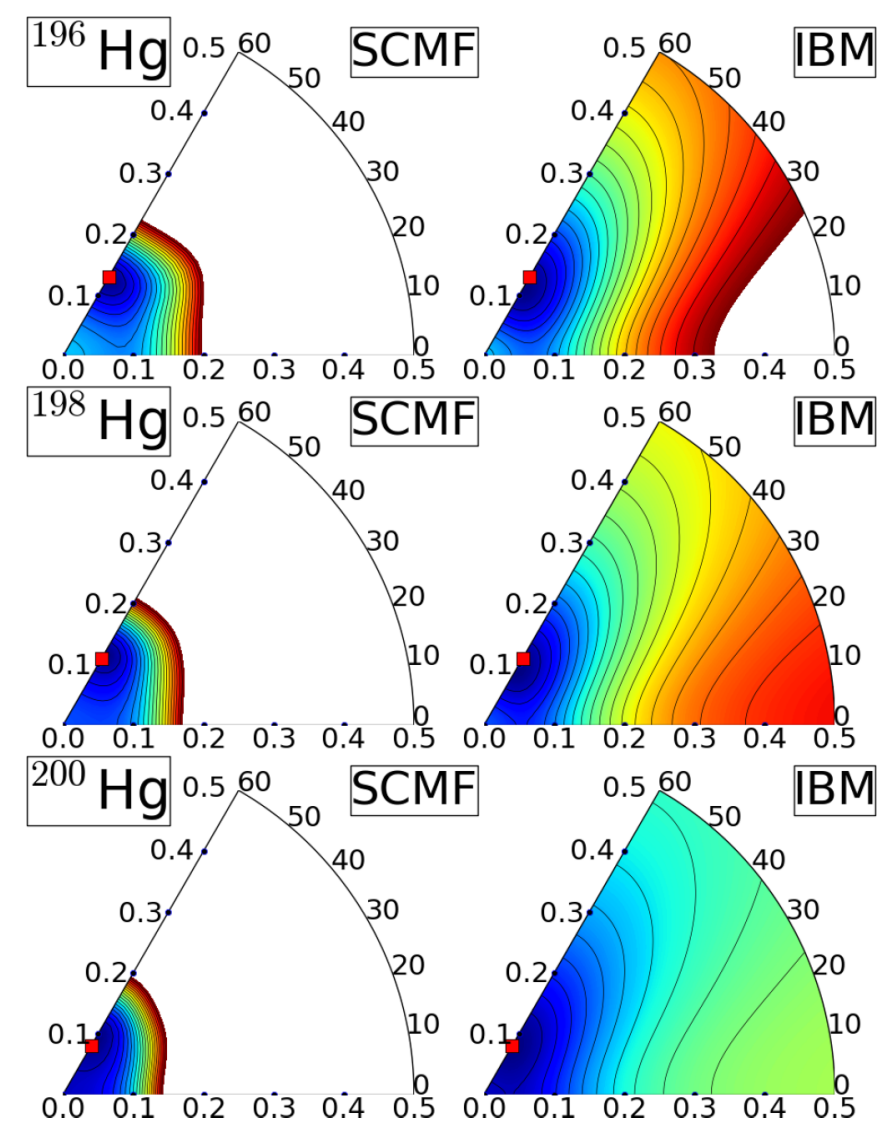

FIG. 1. The Gogny-D1M HFB and mapped IBM-2 energy surfaces in the $(\beta, \gamma)$-deformation space for the ${ }^{196-200} \mathrm{Hg}$ nuclei are plotted up to $5 \mathrm{MeV}$ from the global minimum. The energy difference between the neighboring contours is $250 \mathrm{keV}$. The global minimum is indicated by a filled square.

(see Table III). The calculation reproduces nicely the groundstate band built on the $13 / 2_{1}^{+}$state, which follows the $\Delta I=$ 2 systematic of the weak-coupling limit. For the ${ }^{195,197} \mathrm{Hg}$ nuclei, the calculation suggests that the negative-parity yrast states near the ground state are based mainly on the odd neutron in the $3 p_{1 / 2}$ single-particle orbital coupled to the IBM-2 core. In the case of the nucleus ${ }^{199} \mathrm{Hg}$, however, in most of the yrast states in the vicinity of the ground state three configurations $3 p_{1 / 2}, 3 p_{3 / 2}$, and $2 f_{5 / 2}$ are more strongly mixed than in ${ }^{195,197} \mathrm{Hg}$. Such a change in the structure of the

TABLE VI. Neutron and proton single-particle energies (in $\mathrm{MeV}$ ) and occupation probabilities used in the present study for the odd-odd nuclei ${ }^{194,196,198} \mathrm{Au}$.

\begin{tabular}{cccccccccccccc}
\hline \hline & & $3 p_{1 / 2}$ & $3 p_{3 / 2}$ & $2 f_{5 / 2}$ & $2 f_{7 / 2}$ & $1 h_{9 / 2}$ & $1 i_{13 / 2}$ & & $3 s_{1 / 2}$ & $2 d_{3 / 2}$ & $2 d_{5 / 2}$ & $1 g_{7 / 2}$ & $1 h_{11 / 2}$ \\
\hline${ }^{194} \mathrm{Au}$ & $\epsilon_{j_{v}}$ & 0.000 & 0.913 & 1.013 & 3.804 & 4.238 & 1.502 & $\epsilon_{j_{\pi}}$ & 0.000 & 0.915 & 2.640 & 5.165 & 0.840 \\
& $v_{j_{v}}^{2}$ & 0.254 & 0.521 & 0.555 & 0.945 & 0.950 & 0.699 & $v_{j_{\pi}}^{2}$ & 0.617 & 0.871 & 0.969 & 0.989 & 0.864 \\
${ }^{196} \mathrm{Au}$ & $\epsilon_{j_{v}}$ & 0.000 & 0.929 & 1.036 & 3.831 & 4.321 & 1.535 & $\epsilon_{j_{\pi}}$ & 0.000 & 0.898 & 2.608 & 5.159 & 0.838 \\
& $v_{j_{v}}^{2}$ & 0.296 & 0.595 & 0.632 & 0.956 & 0.962 & 0.767 & $v_{j_{\pi}}^{2}$ & 0.618 & 0.869 & 0.968 & 0.989 & 0.864 \\
${ }^{198} \mathrm{Au}$ & $\epsilon_{j_{v}}$ & 0.000 & 0.949 & 1.059 & 3.861 & 4.405 & 1.570 & $\epsilon_{j_{\pi}}$ & 0.000 & 0.877 & 2.575 & 5.145 & 0.827 \\
& $v_{j_{v}}^{2}$ & 0.346 & 0.675 & 0.714 & 0.967 & 0.972 & 0.831 & $v_{j_{\pi}}^{2}$ & 0.621 & 0.868 & 0.968 & 0.989 & 0.865 \\
\hline \hline
\end{tabular}



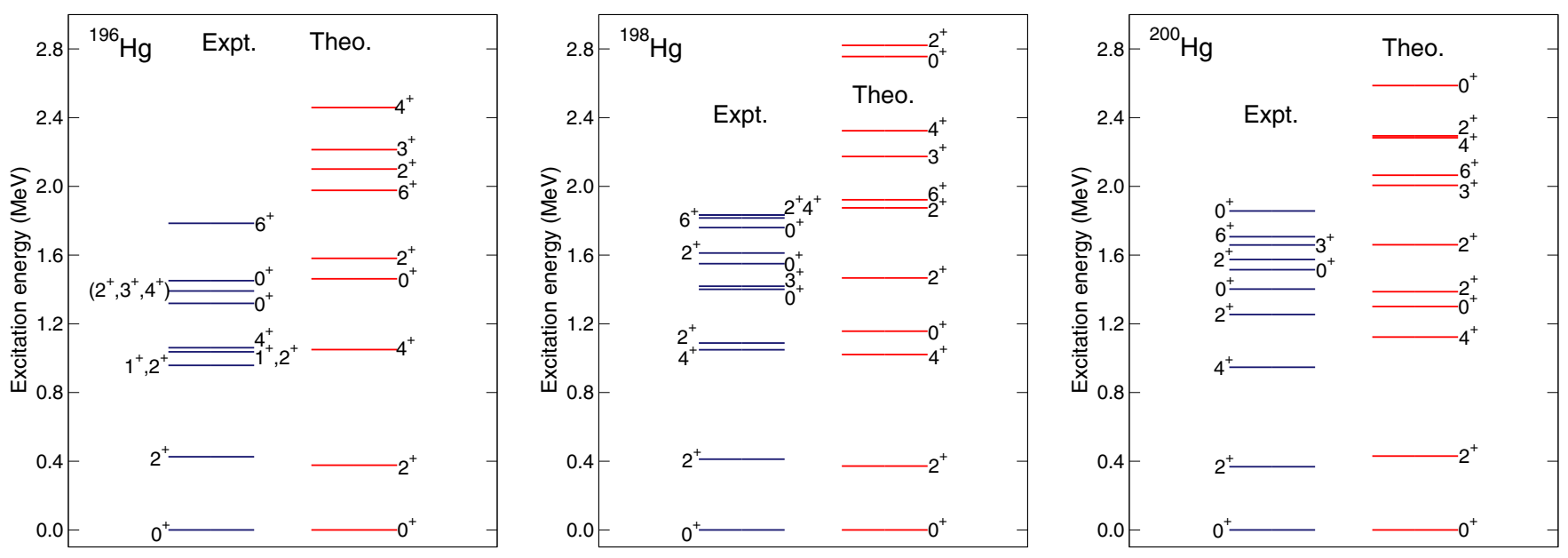

FIG. 2. Theoretical and experimental low-energy excitation spectra for the even-even nuclei ${ }^{196,198,200} \mathrm{Hg}$. The experimental values are taken from the NNDC compilation [53].

low-lying state from ${ }^{195,197} \mathrm{Hg}$ to ${ }^{199} \mathrm{Hg}$ reflects the evolution of shapes in the corresponding even-even systems from ${ }^{198} \mathrm{Hg}$ (weakly oblate deformed) to ${ }^{200} \mathrm{Hg}$ (nearly spherical).

In Fig. 4 we show similar plots for the odd- $Z$ isotopes ${ }^{195,197,199} \mathrm{Au}$. In general, our calculation is in a very good agreement with the experimental data. Our calculation suggests that the IBFM-2 wave functions of the lowest positiveparity states for the considered odd- $Z$ Au nuclei are composed, with a probability of more than $80 \%$, of the $3 s_{1 / 2}$ and $2 d_{3 / 2}$ single-particle configurations, which are substantially mixed with each other. On the other hand, the $2 d_{5 / 2}$ and $1 g_{7 / 2}$ configurations turn out to play minor roles in describing the lowest-lying states.

We confirm that both the $E 2$ and $M 1$ properties of the considered odd-mass nuclei are reasonably described with the present approach.

\section{Odd-odd Au isotopes}

Let us now focus on the discussion of the results for the odd-odd nuclei. The low-lying spectra computed with the IBFFM-2 for the odd-odd ${ }^{194,196,198}$ Au nuclei are depicted in Fig. 5, and compared with the experimental data [53].

$$
\text { 1. }{ }^{194} \mathrm{Au}
$$

First, we observe that the present IBFFM-2 result for the ${ }^{194} \mathrm{Au}$ nucleus is in a very good agreement with the experimental spectra, especially for the positive-parity states. The main component $(\approx 72 \%)$ in the wave functions of the lowest three positive-parity states, i.e., $5_{1}^{+}, 7_{1}^{+}$, and $6_{1}^{+}$ states, is the $\left(v p_{1 / 2} \otimes \pi h_{11 / 2}\right)$ neutron-proton pair coupled to the boson core. For the negative parity, the energy levels near the $1^{-}$ground state are reasonably reproduced in the present calculation. The main components of IBFFM-2 wave function of the $1^{-}$state are the $\left(\nu p_{1 / 2} \otimes \pi s_{1 / 2}\right)_{J=1^{-}}(17 \%)$ and $\left(v f_{5 / 2} \otimes \pi s_{1 / 2}\right)_{J=3^{-}}$neutron-proton pairs $(13 \%)$. However, the calculation is not able to reproduce the experimental $11^{-}$, $12^{-}$, and $13^{-}$levels, which are below $1 \mathrm{MeV}$ excitation. The excitation energies for these states are predicted to be much larger (> $3 \mathrm{MeV}$ ). Empirically, these higher-spin negative parity states are mainly made of the pair composed of the unique-parity orbitals, i.e., $\left(v i_{13 / 2} \otimes \pi h_{11 / 2}\right)$ [53]. The corresponding IBFFM-2 wave functions obtained in the present work are, however, made of the coupling between the odd neutron and proton in the normal-parity orbitals: for instance, the main components of the predicted $11_{1}^{-}$states are the $\left(v p_{1 / 2} \otimes \pi s_{1 / 2}\right)_{J=1^{-}}(35 \%),\left(v f_{5 / 2} \otimes \pi s_{1 / 2}\right)_{J=3^{-}}(19 \%)$, and $\left(v p_{1 / 2} \otimes \pi d_{5 / 2}\right)_{J=3^{-}}(13 \%)$ neutron-protons pairs.

The experimental information about the electromagnetic properties is rather scarce for ${ }^{194} \mathrm{Au}$. Nevertheless, we show in Table VII the calculated $B(E 2)$ and $B(M 1)$ transition rates and quadrupole $Q(I)$ and magnetic $\mu(I)$ moments in comparison with the available data. The predicted $B(E 2)$ values seem to be qualitatively in a good agreement with the data. The calculated $B(M 1)$ values are, however, too large as compared to the experimental values. The sign of the predicted $Q\left(1^{-}\right)$moment is opposite to that given by the experiment.

TABLE VII. Calculated and experimental $B(E 2)$ and $B(M 1)$ transition rates (in Weisskopf units), and quadrupole $Q(I)$ (in $e \mathrm{~b}$ units) and magnetic $\mu(I)$ (in $\mu_{N}$ units) moments for the odd-odd nucleus ${ }^{194} \mathrm{Au}$. The experimental values are taken from Ref. [53].

\begin{tabular}{lcc}
\hline \hline & Theory & Experiment \\
\hline$B\left(E 2 ; 6_{1}^{+} \rightarrow 5_{1}^{+}\right)$ & 3.0 & $5(3)$ \\
$B\left(E 2 ; 7_{1}^{+} \rightarrow 5_{1}^{+}\right)$ & 61 & $27(2)$ \\
$B\left(E 2 ; 8_{1}^{+} \rightarrow 6_{1}^{+}\right)$ & 61 & $22(4)$ \\
$B\left(E 2 ; 8_{1}^{+} \rightarrow 7_{1}^{+}\right)$ & 1.5 & $1.9(5)$ \\
$B\left(M 1 ; 6_{1}^{+} \rightarrow 5_{1}^{+}\right)$ & 0.050 & $0.0010(4)$ \\
$B\left(M 1 ; 6_{1}^{+} \rightarrow 7_{1}^{+}\right)$ & 0.020 & $0.0034(14)$ \\
$B\left(M 1 ; 8_{1}^{+} \rightarrow 7_{1}^{+}\right)$ & 0.021 & $5 \times 10^{-5}(2)$ \\
$Q\left(1_{1}^{-}\right)$ & +0.225 & $-0.240(9)$ \\
$\mu\left(1_{1}^{-}\right)$ & +1.790 & $+0.0763(13)$ \\
\hline \hline
\end{tabular}




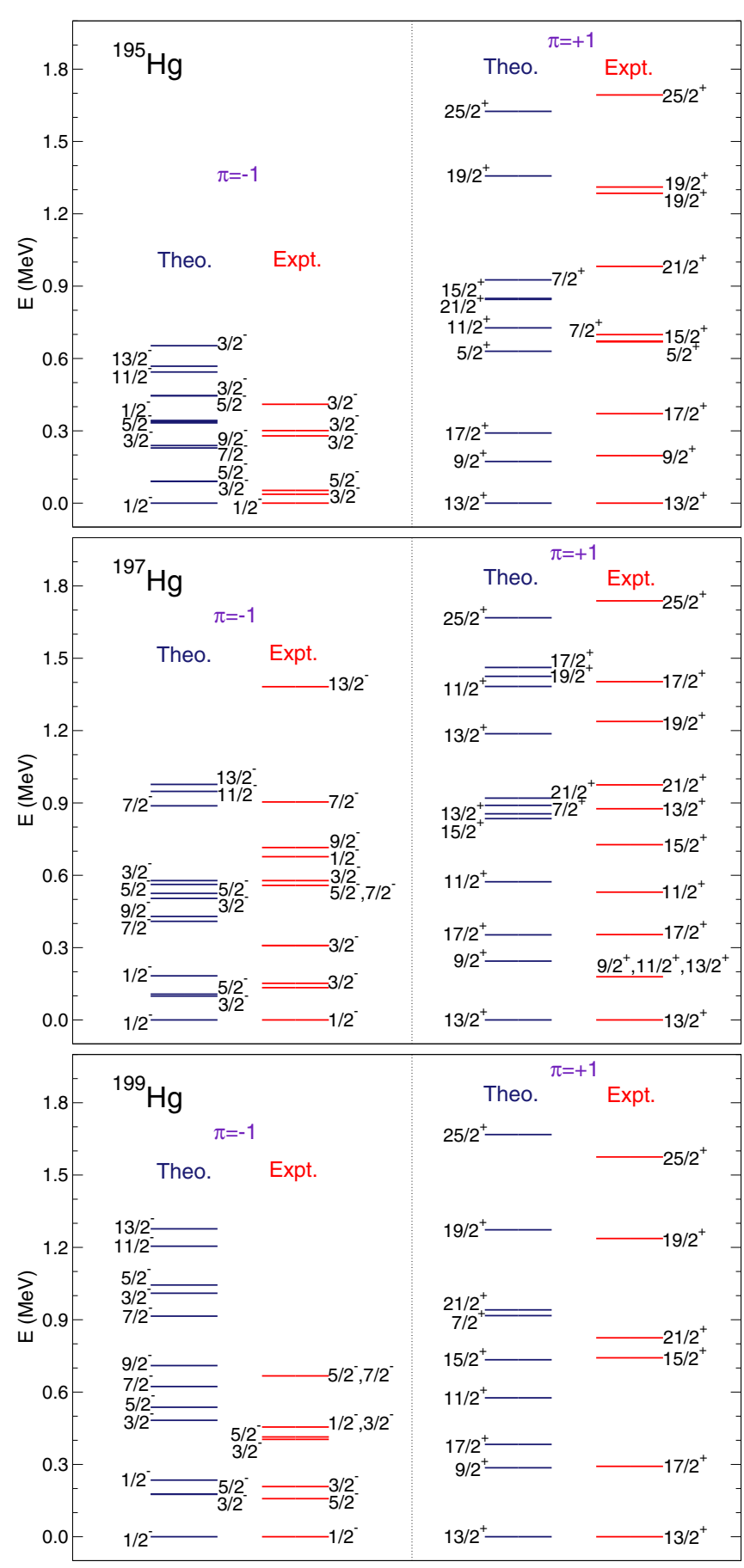

FIG. 3. Same as Fig. 2, but for the odd- $N$ nuclei ${ }^{195,197,199} \mathrm{Hg}$.

\section{2. ${ }^{196} \mathrm{Au}$}

From the comparison of energy levels of the odd-odd nucleus ${ }^{196} \mathrm{Au}$, shown in Fig. 5, one concludes that the calculation is able to reproduce both the experimental positiveand negative-parity levels reasonably well. The calculated low-lying positive-parity states for ${ }^{196} \mathrm{Au}$ are similar in structure to those for ${ }^{194} \mathrm{Au}$ : $67 \%$ and $66 \%$ of the predicted $5_{1}^{+}$ and $7_{1}^{+}$states are dominated by the $\left(v p_{1 / 2} \otimes h_{11 / 2}\right)_{J=5^{+}}$pair component, respectively. The spin of the calculated lowest

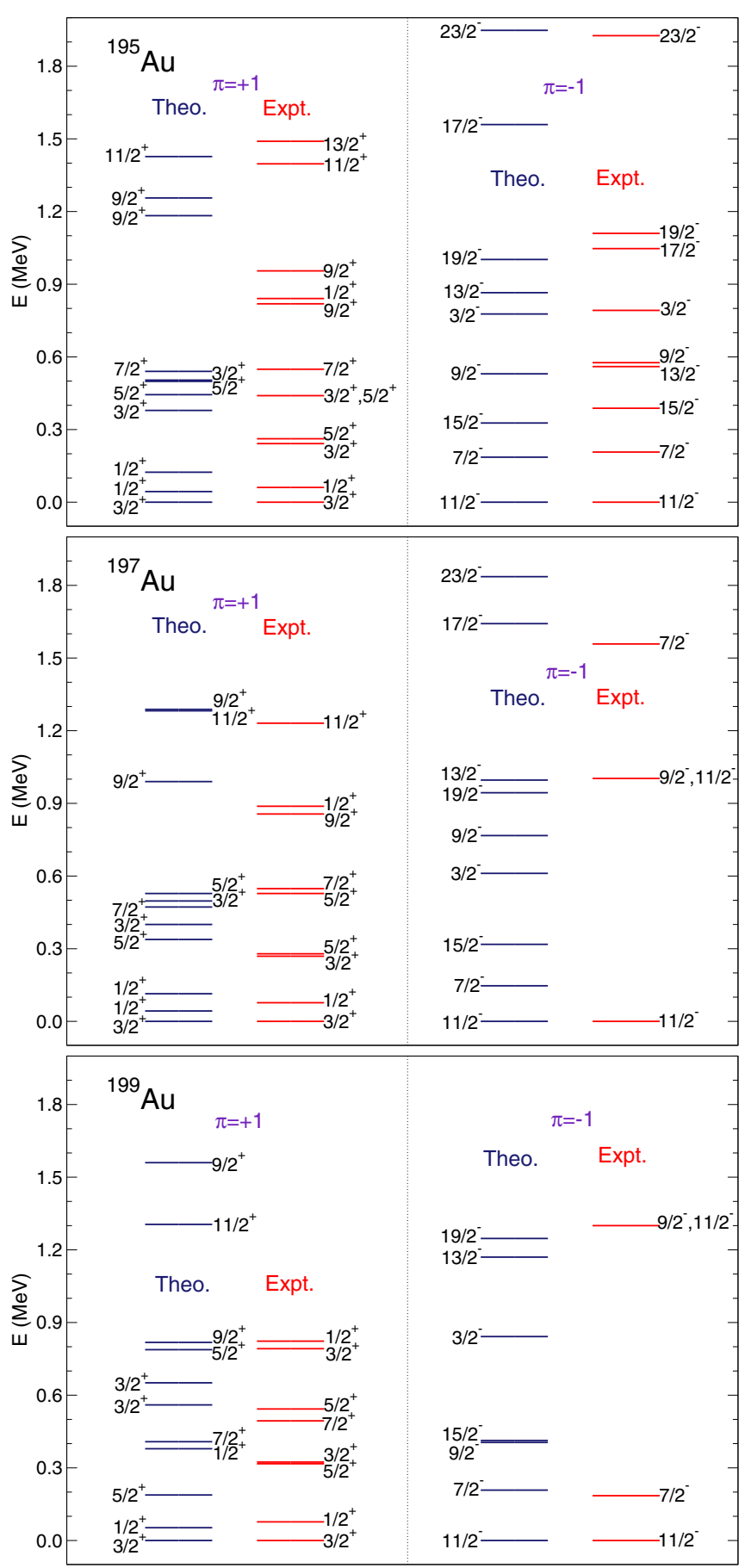

FIG. 4. Same as Fig. 2, but for the odd-Z nuclei ${ }^{195,197,199} \mathrm{Au}$.

negative parity state is $I=1^{-}$. This is at variance with the experiment, although the experimental $1_{1}^{-}$level is only $6 \mathrm{keV}$ above the $2_{1}^{-}$ground state. Furthermore, the present calculation considerably overestimates the $2_{1}^{-}$energy level. The non-negligible components $(>10 \%)$ of the corresponding IBFFM-2 wave functions for the $2_{1}^{-}$and $1_{1}^{-}$states are the following: $\left(v p_{1 / 2} \otimes \pi d_{3 / 2}\right)_{J=2^{-}}(24 \%),\left(v p_{1 / 2} \otimes \pi s_{1 / 2}\right)_{J=1^{-}}$ $(11 \%)$, and $\left(v f_{5 / 2} \otimes \pi s_{1 / 2}\right)_{J=2^{-}}(11 \%)$ for the $2_{1}^{-}$state, and $\left(v p_{1 / 2} \otimes \pi s_{1 / 2}\right)_{J=1^{-}}(38 \%)$ and $\left(v p_{1 / 2} \otimes \pi d_{3 / 2}\right)_{J=1^{-}}(10 \%)$ for the $1_{1}^{-}$state. 

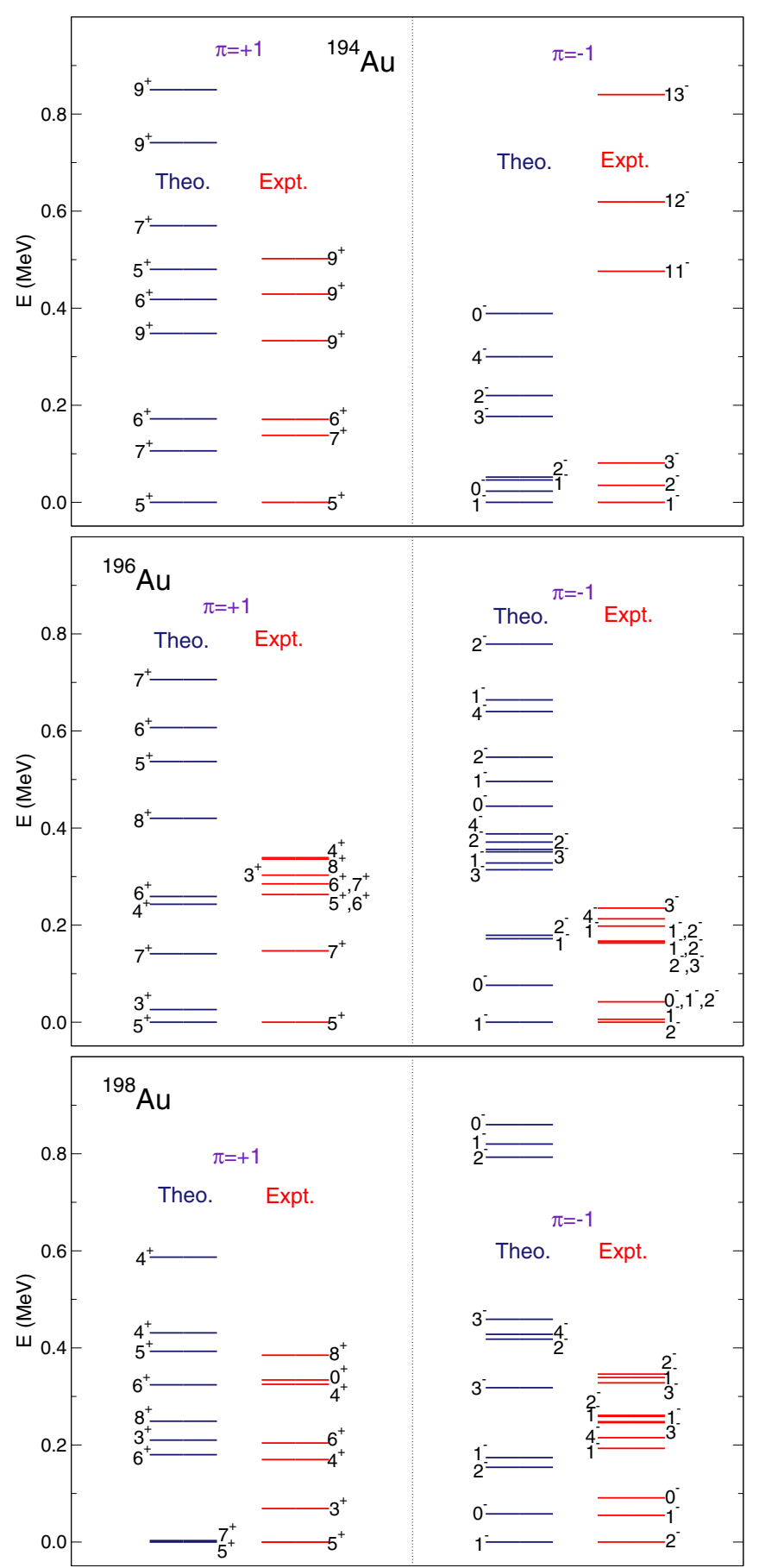

FIG. 5. Same as Fig. 2, but for the odd-odd nuclei ${ }^{194,196,198} \mathrm{Au}$. The experimental values are taken from Refs. [53,54] (for ${ }^{194} \mathrm{Au}$ ), [53,55] (for ${ }^{196} \mathrm{Au}$ ), and [53] (for ${ }^{198} \mathrm{Au}$ ).

Table VIII exhibits the calculated and experimental electromagnetic properties. Regarding the $B(E 2)$ rates, our results are in a reasonable agreement with the experiment. However, similarly to ${ }^{196} \mathrm{Au}$ the calculated $B(M 1)$ values are generally much larger than the experimental values. A number of experimental $B(E 2)$ and $B(M 1)$ transition rates from the $1^{-}$state at the excitation energy of $E_{\mathrm{x}}=298.5 \mathrm{keV}$ are available [55]. However, there are also too many experimental $1^{-}$states
TABLE VIII. Same as Table VII, but for the nucleus ${ }^{196} \mathrm{Au}$. The experimental values are taken from Refs. [53,55].

\begin{tabular}{lcc}
\hline \hline & Theory & Experiment \\
\hline$B\left(E 2 ; 6_{1}^{+} \rightarrow 5_{1}^{+}\right)$ & 2.1 & $>0.064$ \\
$B\left(E 2 ; 6_{2}^{+} \rightarrow 5_{1}^{+}\right)$ & 7.7 & $>0.0068$ \\
$B\left(E 2 ; 7_{1}^{+} \rightarrow 5_{1}^{+}\right)$ & 50 & $51(6)$ \\
$B\left(E 2 ; 7_{2}^{+} \rightarrow 5_{1}^{+}\right)$ & 0.24 & $>0.064$ \\
$B\left(E 2 ; 8_{1}^{+} \rightarrow 6_{1}^{+}\right)$ & 51 & $0.77(39)$ \\
$B\left(E 2 ; 8_{1}^{+} \rightarrow 6_{2}^{+}\right)$ & 0.28 & $20(7)$ \\
$B\left(E 2 ; 8_{1}^{+} \rightarrow 7_{1}^{+}\right)$ & 1.0 & $0.76 \times 10^{-1}(26)$ \\
$B\left(E 2 ; 8_{1}^{+} \rightarrow 7_{2}^{+}\right)$ & 0.018 & $0.77(39)$ \\
$B\left(E 2 ; 3_{2}^{-} \rightarrow 1_{1}^{-}\right)$ & 11 & $>6.5$ \\
$B\left(E 2 ; 4_{1}^{-} \rightarrow 2_{1}^{-}\right)$ & 21 & $9.7(2.4)$ \\
$B\left(E 2 ; 4_{1}^{-} \rightarrow 2_{2}^{-}\right)$ & 1.8 & $13.2(13.2)$ \\
$B\left(E 2 ; 4_{1}^{-} \rightarrow 3_{1}^{-}\right)$ & 0.34 & $13.2(13.2)$ \\
$B\left(M 1 ; 6_{1}^{+} \rightarrow 7_{1}^{+}\right)$ & 0.029 & $3.5 \times 10^{-5}$ \\
$B\left(M 1 ; 6_{2}^{+} \rightarrow 6_{1}^{+}\right)$ & 0.077 & $>0.00016$ \\
$B\left(M 1 ; 6_{2}^{+} \rightarrow 7_{1}^{+}\right)$ & 0.14 & $3.5 \times 10^{-5}$ \\
$B\left(M 1 ; 6_{2}^{+} \rightarrow 7_{2}^{+}\right)$ & 0.050 & $>0.00016$ \\
$B\left(M 1 ; 7_{2}^{+} \rightarrow 7_{1}^{+}\right)$ & 0.0053 & $3.5 \times 10^{-5}$ \\
$B\left(M 1 ; 8_{1}^{+} \rightarrow 7_{1}^{+}\right)$ & 0.022 & $>.49 \times 10^{-3}(5)$ \\
$B\left(M 1 ; 3_{2}^{-} \rightarrow 2_{1}^{-}\right)$ & 0.17 & $>0.0045$ \\
$Q(2-)$ & +0.495 & $0.81(7)$ \\
$\mu\left(22_{1}^{-}\right)$ & +0.197 & $+0.580(15)$ \\
\hline \hline
\end{tabular}

below $298.5 \mathrm{keV}$, and it is not clear which theoretical $1^{-}$state corresponds to the experimental one observed at $E_{\mathrm{x}}=298.5$ $\mathrm{keV}$. For this reason, we do not compare our results with the experimental $B(E 2)$ and $B(M 1)$ transitions rates from the $1^{-}(298.5 \mathrm{keV})$ state.

\section{3. ${ }^{198} \mathrm{Au}$}

As one sees from the comparison between the theoretical and experimental low-energy spectra for the odd-odd nucleus ${ }^{198} \mathrm{Au}$ in Fig. 5, the description of the positive-parity states is generally good. As in the case of ${ }^{196} \mathrm{Au}$, however, our calculation fails to reproduce the spin of the lowest negative-parity state. The structure of the $2_{1}^{-}$and $1_{1}^{-}$wave functions for ${ }^{198} \mathrm{Au}$ turn out to be rather similar to those of ${ }^{196} \mathrm{Au}$, that is, $\left(v p_{1 / 2} \otimes\right.$ $\left.\pi d_{3 / 2}\right)_{J=2^{-}}(26 \%),\left(v p_{1 / 2} \otimes \pi s_{1 / 2}\right)_{J=1^{-}}(19 \%)$, and $\left(v p_{1 / 2} \otimes\right.$ $\left.\pi s_{1 / 2}\right)_{J=0^{-}}(12 \%)$ for the $2_{1}^{-}$state, and $\left(v p_{1 / 2} \otimes \pi s_{1 / 2}\right)_{J=1^{-}}$ $(41 \%)$ and $\left(v p_{1 / 2} \otimes \pi d_{3 / 2}\right)_{J=1^{-}}(13 \%)$ for the $1_{1}^{-}$state. The previous IBFFM calculation of [29] obtains an excellent description of both the positive- and negative-parity levels. The IBFFM wave functions they obtained are predominantly described by the $\left(v p_{1 / 2} \otimes \pi d_{3 / 2}\right)_{J=2^{-}}$component $(>70 \%)$ for the $2_{1}^{-}$state and $\left(v p_{1 / 2} \otimes \pi d_{3 / 2}\right)_{J=1^{-}}(50 \%)$ for the $1_{1}^{-}$state. The difference between our result and that of [29] could be accounted for by the different single-particle energies used in each study. In the present calculation, the $2 d_{3 / 2}$ single-particle orbital is about $0.9 \mathrm{MeV}$ above the $3 s_{1 / 2}$ (see Table VI). On the other hand, in [29] the $2 d_{3 / 2}$ orbital is below the $3 s_{1 / 2}$ one and, consequently, the $\pi d_{3 / 2}$ single-particle configuration plays a more dominant role in low-energy region than in our calculation. 
TABLE IX. Same as Table VII, but for the nucleus ${ }^{198} \mathrm{Au}$.

\begin{tabular}{lll}
\hline \hline & Theory & \multicolumn{1}{c}{ Experiment } \\
\hline$B\left(E 2 ; 1_{2}^{-} \rightarrow 2_{1}^{-}\right)$ & 6.7 & $2.2(7)$ \\
$B\left(E 2 ; 2_{3}^{-} \rightarrow 4_{1}^{-}\right)$ & 0.049 & $>64$ \\
$B\left(E 2 ; 3_{1}^{-} \rightarrow 1_{1}^{-}\right)$ & 8.3 & $>26$ \\
$B\left(E 2 ; 3_{1}^{-} \rightarrow 2_{1}^{-}\right)$ & 2.1 & $>13$ \\
$B\left(E 2 ; 4_{1}^{-} \rightarrow 2_{1}^{-}\right)$ & 13 & $35(18)$ \\
$B\left(M 1 ; 1_{2}^{-} \rightarrow 0_{1}^{-}\right)$ & 0.015 & $0.0032(10)$ \\
$B\left(M 1 ; 1_{2}^{-} \rightarrow 1_{1}^{-}\right)$ & 0.0049 & $0.00024(8)$ \\
$B\left(M 1 ; 1_{3}^{-} \rightarrow 0_{1}^{-}\right)$ & 0.00067 & $7.4 \times 10^{-5}(24)$ \\
$B\left(M 1 ; 1_{3}^{-} \rightarrow 1_{1}^{-}\right)$ & 0.00051 & $0.0017(5)$ \\
$B\left(M 1 ; 1_{4}^{-} \rightarrow 0_{1}^{-}\right)$ & $8.2 \times 10^{-5}$ & $>0.0084$ \\
$B\left(M 1 ; 1_{4}^{-} \rightarrow 2_{1}^{-}\right)$ & 0.0021 & $>9.9 \times 10^{-6}$ \\
$B\left(M 1 ; 1_{5}^{-} \rightarrow 1_{1}^{-}\right)$ & 0.022 & $>0.00029$ \\
$B\left(M 1 ; 1_{5}^{-} \rightarrow 1_{2}^{-}\right)$ & 0.042 & $>0.0042$ \\
$B\left(M 1 ; 1_{6}^{-} \rightarrow 0_{1}^{-}\right)$ & 0.0013 & $>0.00025$ \\
$B\left(M 1 ; 1_{6}^{-} \rightarrow 1_{4}^{-}\right)$ & 0.10 & $>0.015$ \\
$B\left(M 1 ; 1_{6}^{-} \rightarrow 2_{1}^{-}\right)$ & 0.0010 & $>5.6 \times 10^{-5}$ \\
$B\left(M 1 ; 2_{2}^{-} \rightarrow 1_{1}^{-}\right)$ & 0.064 & $>0.00033$ \\
$B\left(M 1 ; 2_{2}^{-} \rightarrow 2_{1}^{-}\right)$ & 0.024 & $>0.0037$ \\
$B\left(M 1 ; 2_{3}^{-} \rightarrow 1_{2}^{-}\right)$ & $4.5 \times 10^{-6}$ & $>0.00065$ \\
$B\left(M 1 ; 2_{3}^{-} \rightarrow 1_{3}^{-}\right)$ & 0.024 & $>0.0048$ \\
$B\left(M 1 ; 2_{3}^{-} \rightarrow 2_{1}^{-}\right)$ & $8.5 \times 10^{-5}$ & $>0.00042$ \\
$B\left(M 1 ; 2_{4}^{-} \rightarrow 1_{4}^{-}\right)$ & 0.0015 & $>0.015$ \\
$B\left(M 1 ; 3_{1}^{-} \rightarrow 2_{1}^{-}\right)$ & 0.42 & $>0.0019$ \\
$B\left(M 1 ; 3_{2}^{-} \rightarrow 2_{1}^{-}\right)$ & 0.0042 & $>0.0026$ \\
$Q(2-$ & +0.373 & $+0.59(2)$ \\
$\mu\left(5_{1}^{+}\right)$ & +4.398 & \\
$\mu(2)$ & +0.334 & \\
\hline \hline
\end{tabular}

In Table IX the calculated $B(E 2)$ values for ${ }^{198} \mathrm{Au}$ are, in general, in good agreement with the experiment. We also present the calculated $B(M 1)$, but for most of the available data only a lower limit for this quantity is known. The calculated magnetic moment of the $5_{1}^{+}$state, $\mu\left(5_{1}^{+}\right)$, has the opposite sign and is a factor of 4 larger in magnitude than the experimental one. Similar results have been obtained for the ${ }^{194,196} \mathrm{Au}$ nuclei as we obtain $\mu\left(5_{1}^{+}\right) \approx 5 \mu_{N}$. As already mentioned, the $5_{1}^{+}$states obtained in the present calculation for the considered odd-odd Au nuclei are dominated by the $\left(v p_{1 / 2} \otimes \pi h_{11 / 2}\right)_{J=5^{+}}$neutron-proton pair configuration, and the predicted $\mu\left(5_{1}^{+}\right)$moments are mostly accounted for by this configuration, in particular, by the odd-proton part of the M1 matrix element, which takes large positive value. On the other hand, empirical studies for the low-lying level structure of ${ }^{194} \mathrm{Au}[54,56]$ assume the $5_{1}^{+}$state and the band built on it to be based mainly on the $\left(\nu i_{13 / 2}^{-1} \otimes \pi d_{3 / 2}^{-1}\right)_{J=5^{+}}$configuration, leading to the correct sign of the $\mu\left(5_{1}^{+}\right)$moment.

\section{SUMMARY AND CONCLUDING REMARKS}

In this work, we extend the recently developed method of Ref. [15] for calculating the spectroscopy of odd-mass nuclei to odd-odd systems. The $(\beta, \gamma)$-deformation energy surfaces of the even-even core nuclei, and spherical single-particle energies and occupation probabilities of the odd neutron and the odd proton, are calculated by the constrained HFB method based on the Gogny D1M EDF. These quantities are then used as microscopic input to build most of the different terms of the IBFFM-2 Hamiltonian. The strength parameters for the boson-fermion interaction terms in the IBFFM-2 Hamiltonian are taken from those of the neighboring odd-mass nuclei. Two coefficients in the residual interaction between odd neutron and proton are the only new parameters, and are determined as to reproduce the low-energy levels of each odd-odd nucleus. In this way, we are able to reduce significantly the number of free parameters in the IBFFM2 framework. It is shown that the method provides a reasonable description of low-energy spectra and electromagnetic properties of the odd-odd nuclei ${ }^{194,196,198} \mathrm{Au}$. Even though a few strength parameters in the boson-fermion and fermion-fermion interactions are treated as free parameters, the method developed in this paper, as well as in Ref. [15], in which the even-even IBM-core Hamiltonian is determined fully microscopically and only one or two unpaired nucleon degrees of freedom are added via the particle-boson coupling, allows for a simultaneous description of a large number of even-even, odd-mass, and odd-odd medium-mass and heavy nuclei.

\section{ACKNOWLEDGMENTS}

This work was supported in part by the QuantiXLie Centre of Excellence, a project cofinanced by the Croatian Government and European Union through the European Regional Development Fund: the Competitiveness and Cohesion Operational Programme (Grant No. KK.01.1.1.01.0004). The work of L.M.R. was supported by Spanish Ministry of Economy and Competitiveness (MINECO) Grants No. FPA2015-65929-MINECO and No. FIS2015-63770MINECO.
[1] A. Bohr and B. M. Mottelsson, Nuclear Structure, Vol. 2 (Benjamin, New York, 1975).

[2] P. Ring and P. Schuck, The Nuclear Many-Body Problem (Springer-Verlag, Berlin, 1980).

[3] F. Iachello and A. Arima, The Interacting Boson Model (Cambridge University Press, Cambridge, 1987).

[4] R. F. Casten, Nuclear Structure from a Simple Perspective (Oxford University Press, Oxford, 1990).
[5] M. Bender, P.-H. Heenen, and P.-G. Reinhard, Rev. Mod. Phys. 75, 121 (2003).

[6] E. Caurier, G. Martínez-Pinedo, F. Nowacki, A. Poves, and A. P. Zuker, Rev. Mod. Phys. 77, 427 (2005).

[7] A. Bohr, Mat. Fys. Medd. K. Dan. Vidensk. Selsk. 27, 16 (1953).

[8] T. Otsuka, A. Arima, and F. Iachello, Nucl. Phys. A 309, 1 (1978). 
[9] T. Otsuka, A. Arima, F. Iachello, and I. Talmi, Phys. Lett. B 76, 139 (1978).

[10] T. Otsuka, Phys. Lett. B 138, 1 (1984).

[11] T. Mizusaki and T. Otsuka, Prog. Theor. Phys. Suppl. 125, 97 (1996).

[12] K. Nomura, N. Shimizu, and T. Otsuka, Phys. Rev. Lett. 101, 142501 (2008).

[13] K. Nomura, T. Otsuka, N. Shimizu, and L. Guo, Phys. Rev. C 83, 041302 (2011).

[14] J. N. Ginocchio and M. W. Kirson, Nucl. Phys. A 350, 31 (1980).

[15] K. Nomura, T. Nikšić, and D. Vretenar, Phys. Rev. C 93, 054305 (2016).

[16] F. Iachello and P. Van Isacker, The Interacting Boson-Fermion Model (Cambridge University Press, Cambridge, 1991).

[17] K. Nomura, T. Nikšić, and D. Vretenar, Phys. Rev. C 94, 064310 (2016).

[18] K. Nomura, T. Nikšić, and D. Vretenar, Phys. Rev. C 96, 014304 (2017).

[19] K. Nomura, R. Rodríguez-Guzmán, and L. M. Robledo, Phys. Rev. C 96, 064316 (2017).

[20] K. Nomura, R. Rodríguez-Guzmán, and L. M. Robledo, Phys. Rev. C 97, 064314 (2018).

[21] K. Nomura, T. Nikšić, and D. Vretenar, Phys. Rev. C 97, 024317 (2018).

[22] K. Nomura, R. Rodríguez-Guzmán, and L. M. Robledo, Phys. Rev. C 97, 064313 (2018).

[23] S. Brant, V. Paar, and D. Vretenar, Z. Phys. A: At. Nucl. 319, 355 (1984).

[24] S. Goriely, S. Hilaire, M. Girod, and S. Péru, Phys. Rev. Lett. 102, 242501 (2009).

[25] J. F. Berger, M. Girod, and D. Gogny, Nucl. Phys. A 428, 23 (1984).

[26] J.-P. Delaroche et al., Phys. Rev. C 81, 014303 (2010); http://www-phynu.cea.fr/science_en_ligne/carte_potentiels_ microscopiques/carte_potentiel_nucleaire_eng.htm\#info

[27] L. M. Robledo and G. F. Bertsch, Phys. Rev. C 84, 054302 (2011).

[28] K. Nomura, R. Rodríguez-Guzmán, and L. M. Robledo, Phys. Rev. C 87, 064313 (2013).

[29] V. Lopac, S. Brant, V. Paar, O. W. B. Schult, H. Seyfarth, and A. B. Balantekin, Z. Phys. A: At. Nucl. 323, 491 (1986).

[30] N. Blasi and G. L. Bianco, Phys. Lett. B 185, 254 (1987).

[31] P. Van Isacker, J. Jolie, K. Heyde, and A. Frank, Phys. Rev. Lett. 54, 653 (1985).

[32] J. Barea, R. Bijker, and A. Frank, Phys. Rev. Lett. 94, 152501 (2005).
[33] T. Thomas, J.-M. Régis, J. Jolie, S. Heinze, M. Albers, C. Bernards, C. Fransen, and D. Radeck, Nucl. Phys. A 925, 96 (2014).

[34] F. Iachello, Phys. Rev. Lett. 44, 772 (1980).

[35] L. M. Robledo, R. Bernard, and G. F. Bertsch, Phys. Rev. C 86, 064313 (2012).

[36] R. Rodríguez-Guzmán, P. Sarriguren, L. M. Robledo, and S. Perez-Martin, Phys. Lett. B 691, 202 (2010).

[37] R. Rodríguez-Guzmán, P. Sarriguren, and L. M. Robledo, Phys. Rev. C 82, 044318 (2010).

[38] R. Rodríguez-Guzmán, P. Sarriguren, and L. M. Robledo, Phys. Rev. C 82, 061302 (2010).

[39] L. M. Robledo, R. N. Bernard, and G. F. Bertsch, Phys. Rev. C 89, 021303 (2014).

[40] J. Dobaczewski, A. Afanasjev, M. Bender, L. Robledo, and Y. Shi, Nucl. Phys. A 944, 388 (2015), Special Issue on Superheavy Elements.

[41] B. Bally, B. Avez, M. Bender, and P.-H. Heenen, Phys. Rev. Lett. 113, 162501 (2014).

[42] M. Borrajo and J. L. Egido, Eur. Phys. J. A 52, 277 (2016).

[43] O. Scholten, Prog. Part. Nucl. Phys. 14, 189 (1985).

[44] J. M. Arias, C. E. Alonso, and M. Lozano, Phys. Rev. C 33, 1482 (1986).

[45] S. Brant, N. Yoshida, and L. Zuffi, Phys. Rev. C 74, 024303 (2006).

[46] N. Yoshida and F. Iachello, Prog. Theor. Exp. Phys. 2013, 043D01 (2013).

[47] I. Morrison, A. Faessler, and C. Lima, Nucl. Phys. A 372, 13 (1981).

[48] R. Bark, J. Espino, W. Reviol, P. Semmes, H. Carlsson, I. Bearden, G. Hagemann, H. Jensen, I. Ragnarsson, L. Riedinger et al., Phys. Lett. B 406, 193 (1997).

[49] D. J. Thouless and J. G. Valatin, Nucl. Phys. 31, 211 (1962).

[50] K. Nomura, R. Rodríguez-Guzmán, and L. M. Robledo, Phys. Rev. C 96, 014314 (2017).

[51] N. Yoshida (private communucation).

[52] N. Yoshida and A. Arima, Phys. Lett. B 164, 231 (1985).

[53] Brookhaven National Nuclear Data Center, http://www.nndc. bnl.gov.

[54] B. S. Gao, X. H. Zhou, Y. D. Fang, Y. H. Zhang, S. C. Wang, N. T. Zhang, M. L. Liu, J. G. Wang, F. Ma, Y. X. Guo et al., Phys. Rev. C 86, 054310 (2012).

[55] P. Petkov, J. Jolie, S. Heinze, S. Drissi, M. Dorthe, J. Gröger, and J. Schenker, Nucl. Phys. A 796, 1 (2007).

[56] A. Pakkanen, M. Piiparinen, M. Kortelahti, T. Komppa, R. Komu, and J. Äystö, Z. Phys. A: At. Nucl. 282, 277 (1977). 